\title{
Learner Mathematics Learning Beliefs System: A Case Study
}

\author{
* Ms. Ramim Bibi, PhD Scholar \\ ** Dr. Amir Zaman, Assistant Professor \\ *** Dr. Muhammad Idris, Assistant Professor
}

\begin{abstract}
This study makes an effort to investigate learner mathematics-related beliefs at the secondary level. The study was qualitative and the method adopted for the in-depth information about the belief was a single case study. The site were selected for this investigation were Government Girls Higher Secondary School Rustam in District Mardan. The number of participants was six from class $10^{\text {th }}$ science students. In-depth interviews were scheduled for the collection of data. Interview items were 9 in number. Thematic analysis of the transcripts exposed learner beliefs about mathematics. The main findings of the study showed that those students who understand the concept of mathematics possessed the positive beliefs about mathematics, and they also like the book and course materials and satisfied with it. They feel easy during mathematics classrooms, enjoy, and excited to learn something new in this subject. In contrast, the learners who do not understanding level is low they hold negative beliefs they did not like the book and course materials and not satisfied and feel hesitation during mathematics classroom. The study further showed that in the construction of beliefs that are positive or negative about the mathematics classroom environment play a great role especially mathematics teacher, her methods, and her beliefs. If the teacher involves the students in activities, keep good and interesting method and motivate the students time to time, then the learners will possess positive beliefs and will take interest in such a very important mathematics class.
\end{abstract}

Keywords: Mathematics; Learning; Learners; Belief System

Introduction

Education is the achievement of knowledge, skills, values, beliefs, and habits under the guidance of educators. Mathematics education is referred to as the practice of teaching and learning of mathematics in a way of solving problems involving learning the algorithms and formulas necessary for computations. Hadi (2017) studied that knowledge about every subject is important but knowledge of mathematics is the most powerful tool in the world. Beliefs are defined as "knowledge" constructed by the individual. This "knowledge" is not provable by others and the truthfulness of this "knowledge" is only self-evident within the individual (Op'tEynde et al, 2002). Callejo and Vila (2009) recognize beliefs as a learner's one-sided knowledge of an exact mathematical content, him/ herself as a mathematics learner, and mathematics problem-solving. Beliefs play a great role in mathematics learning and teaching. The learning outcomes of students are strongly related to their beliefs and attitudes about mathematics (Furinghetti \& Pehkonen, 2000). Mathematical-related beliefs are important to study because beliefs can be used to explain students' behaviours in numerous mathematical settings Schoenfeld (2015) argued that to fully understand students' mathematical performance researchers need to understand students' mathematics-related beliefs including beliefs about self, mathematical tasks, social context of mathematical learning, facts, problem-solving procedures, and strategies.

Op'tEynde and colleagues (2002) stated that 'students' mathematics-related beliefs are completely or held skewed conceptions students hold to be true, that influence their mathematical learning and problem solving". Physick (2010) conducted their study the result supported the belief that self-confidence has a strong correlation with achievement in mathematics. Hannula et al (2005) divided learners' beliefs into three groups the first was a positive core belief the second was negative core belief and the third one was a neutral core belief. Their negative belief was that they work hard but not succeed and also they cannot learn mathematics. Chirove (2014) inspected that the learner belief systems initiate from their social context (classroom environment) and provide their individual

\footnotetext{
* Department of Education, Abdul Wali Khan University Mardan Email: ayatulahraas03204@gmail.com

** Department of Education, Abdul Wali Khan University Mardan Email: dr.amirzaman@awkum.edu.pk

*** Department of Education, Abdul Wali Khan University Mardan Email: midrees@awkum.edu.pk
} 
needs such as emotional need, social need, and their interest. The researcher concluded that the learner's belief systems construct from four things that is the nature of mathematics, teacher role, the learner itself, and from the mathematics classroom.

\section{Statement of the Problem}

This study determines 'learner Mathematics related belief system at the Secondary level.

Objectives of the Study

The objective of the study was:

1. To investigate learners' Mathematics related belief systems at the Secondary level.

\section{Research Questions}

1. What is the grade $10^{\text {th }}$ learner's mathematics-related belief systems at the Secondary level?

\section{Significance of the Study}

This study added new knowledge about students' mathematics-related beliefs and provided information to mathematics instructors to better understand how students' mathematics-related beliefs will affect the implementation of Mathematical modeling and students' problem-solving abilities. The teacher will be aware of their learner's strong and weak areas in learning of mathematics. The result of this study will reduce the gap between theory and practice in mathematics education. The researcher will suggest teaching and learning methods that develop healthy mathematical beliefs.

\section{Literature Review}

Education is the achievement of knowledge, skills, values, beliefs, and habits under the guidance of educators. Mathematics is a symbolic language that can be used in science. Mathematics is a language that can be used in communication (Armianti, 2009). In all around the world, Mathematics is an important part of the curriculum. In Pakistan, mathematics is a compulsory subject at all school levels because the knowledge of mathematics a vital for most of the subjects (Akhter, 2018). Bishop (2014) studied the importance of mathematics in the present situation. He said that in the modern world mathematics is one the most important subjects at school but unfortunately, it is a difficult subject for most learners as well as for the teachers. Tambunan (2018) studying mathematics means knowledge to solve problems, both problems related to everyday problems as well as solving mathematical problems themselves. Saadeq (2019) studied that due to lack of motivation, the students do not taking interest and often weak in mathematics and possess negative beliefs that are, mathematics is a boring and difficult subject which is the cause of their failure in mathematics. The researcher explains that at the primary level $2.3 \%$ of students could not perform well in mathematics but $1.1 \%$ for secondary students. Callejo and Vila (2009) recognize beliefs as a learner's one-sided knowledge of an exact mathematical content, him/ herself as a mathematics learner, and mathematics problem-solving. Smith (2014) conducted a study on 'How teacher beliefs affect the learners' beliefs".In this study the researcher indicates that beliefs are cognitive and are naturally difficult to change because the mind logically adopts observation to confirm the existing beliefs, instead of changing beliefs to support the new remarks. Beliefs are conscious or unconscious opinions and views of the individual about himself, about the world, or his place in the world. These opinions extend from joining to different social groups and also they are measured as correct by the individual. (Umit \& Celik,2017). Goldin (2002) defines a belief system as a structurally organized set of beliefs that is socially or culturally shared. Op 'tEynde et al. (2006) define learners' mathematics-related belief systems as composed of a learner's consciously or unconsciously her subjective conceptions about mathematics as a subject, about him/herself as a learner of mathematics and about the mathematics classroom context.

Physick (2010) conducted a study on the belief system. The title of his study was "Exploring mathematics-related belief systems. The primary goal of this study was to obtain insight into the mathematically related belief systems that form students' mathematical nature. Giovanni and Sangcap's (2010) view that learner's beliefs and attitudes about mathematics teaching and learning play a great role in mathematics education. Student's achievements are strongly related to their beliefs and attitudes towards mathematics. Aksu (2012) argues that beliefs come from experiences that are gain from the classroom environment. To change learner beliefs the teacher of mathematics change their teaching method because the basic root of beliefs is the classroom environment especially teacher methodology. Beliefs of learner derived from his/her liking and disliking of mathematics. An individual view of mathematics is a mixture of knowledge, belief, conception, attitude, and feelings (Hannula, 2007). 
Maiorca (2016) researched to examine two broad categories of mathematically related belief systems. The first one was beliefs about the nature of mathematics while the second one was beliefs about the self as a learner of mathematics. Levy et al. (2012) suppose that the students develop their belief systems from their epistemological, mental, and common needs. For example the epistemological need is to understanding and clearing up mathematical concepts, facts, theories, and procedures. Psychological or mental needs mean the learner's self-respect and the common or social need mean the learner has a social relationship with their family, with teachers and class fellow in their school. This discussion showed that the learner adopt their belief system from their given environment and their required needs. Kıbrıslığlu (2016) framework had three main categories of beliefs about: (i) mathematics education, (ii) the self and (iii) social context. (Yuanita et al, 2018) presented four broad categories that are beliefs about mathematics, Beliefs about learning of mathematics, Beliefs about the teaching of mathematics' beliefs about mathematics and beliefs about social context. The mathematical belief of students consists of the effectiveness of the Realistic Mathematics Education approach of three main factors, namely, students' belief in their ability, in the mathematical discipline, and towards mathematical teaching and learning.

\section{Theoretical Framework}

The theories that conduct, maintain, and inform this study of research were mathematics-related belief theories (foundations, coherence, and complexity theories). In the current study, the researcher talks about the theories because "all belief systems are based on theories and

Ideologies" (Sorochan, 2011, p. 2); whereas beliefs drive learners' behaviour in non-routine mathematics problem solving (Levy, West \& Rosenthal, 2012).

\section{Mathematics Related Belief Theories}

All belief systems are based on theories and ideologies. There are three theories related to mathematics that is foundation mathematics-related belief theory, coherence mathematics-related belief theory, and complexities theory.

\section{Foundations Mathematics - Related Belief Theory}

The foundations of mathematics-related theory assert that a learner admits or holds beliefs that he/she has justification for their survival. The learners have to hold a visible record of the justification for his/her Mathematics related belief. Furthermore, the researcher claims that if the belief is justified by numerous independent beliefs then the learner holds the belief with removing some justifying belief (Chirove, 2014).

\section{Coherence Mathematics - Related Belief Theory}

There is a contradiction between foundation mathematics theory and coherence theory of mathematics because for foundation theory justification is required and for coherence theory justification is not required but is justified just as they are. All the components of belief systems are logically interconnected with each other.

\section{Complexity Theory of Mathematics - Related Belief Systems}

The theory that processes having a large number of apparently sovereign agents can suddenly order themselves into a coherent system. In a complex system the interchange components are the ability to create a new quality of combined behaviour through self-union. Complexity theory outlook about a system that a system constitutes different interconnected units. The interlink of the unit in a system is the product of the change in the system. It showed that the system is not static it is a dynamic. This theory highlights the non-linear relationship that exists between dynamic systems. Besides, the researcher explains that in a system a simple connection of unit some time generates complex behaviours. A system could be better understood if one traces the relationship between its components.

In the light of Beswick (2011) study the belief systems, it is likely that one belief might be linked with other beliefs, even with other beliefs that are situated in another diverse group, to such an extent that contradictory beliefs might be held by one person; and the relationship between behaviour and a specific belief system is difficult to identify. 
Table1. Ernest's (1991) five ideologies of mathematics education.

(Adopted from Chirove, 2014, (p. 70).

\begin{tabular}{|c|c|c|c|}
\hline & Mathematic & Mathematic learning & Mathematics Teaching \\
\hline Industrial trainer & Following rules & $\begin{array}{l}\text { Hard work, trial and } \\
\text { error, practice, rote }\end{array}$ & $\begin{array}{l}\text { Authoritarian } \\
\text { transmission, drill, no } \\
\text { 'frills' }\end{array}$ \\
\hline Technological pragmatist & $\begin{array}{l}\text { Unquestioned body of } \\
\text { useful knowledge }\end{array}$ & $\begin{array}{l}\text { Skill acquirement, life } \\
\text { experience }\end{array}$ & $\begin{array}{l}\text { Skill teacher, proper } \\
\text { motivation through } \\
\text { work application }\end{array}$ \\
\hline Old humanist & $\begin{array}{l}\text { Body of structured pure } \\
\text { knowledge }\end{array}$ & $\begin{array}{l}\text { Understanding } \\
\text { application }\end{array}$ & $\begin{array}{l}\text { Explain, motivate pass } \\
\text { on structure }\end{array}$ \\
\hline Public Educator & $\begin{array}{l}\text { Personalized } \\
\text { Mathematics }\end{array}$ & $\begin{array}{l}\text { Activity, } \\
\text { exploration }\end{array}$ & $\begin{array}{lr}\begin{array}{l}\text { Facilitate } \\
\text { exploration, } \\
\text { failure }\end{array} & \text { personal } \\
\end{array}$ \\
\hline Public Educator & Social constructivism & $\begin{array}{l}\text { Questioning, decision } \\
\text { making, negotiation }\end{array}$ & $\begin{array}{l}\text { argument, clash, } \\
\text { questioning of content } \\
\& \text { teaching methods }\end{array}$ \\
\hline
\end{tabular}

In short the learner classified into industrial trainer are beliefs that learning mathematics following rules, practice, and memorization is important. The technological pragmatist view that mathematics should be composed of daily life experiences. The old humanistic students view that for learning mathematics teachers play a great role. Progressive educator beliefs on discussion, group work, and cooperation. The belief that mathematics can learn better if there is a discussion session and group work in the classroom.

\section{Methodology}

The nature of the study was qualitative. A single case study research design was employed to obtain in-depth information about learner's mathematics belief systems. (Creswell, 2009).

Case study design is selected because case study design is particularly suited to the study of complex phenomena in which the variables being studied cannot be separated from the context in which they exist (Yin, 2009)

\section{Selection of Participant and Data Collection}

Merriam (1988) asserts that cases "should be selected for their power both to maximize and to minimize differences in the phenomenon of interest" (p. 154). Therefore, the researcher set out to engage participants from a single grade (science students of grade $10^{\text {th }}$ ) to facilitate comparison across individual cases. The number of the participants from which the researcher collect data was six. The site selected for the research was Government Girls Higher Secondary School Rustam Mardan to participate in this study.

\section{Tool for Investigation}

In-depth Interview was constructed for the collection of data. The interview schedule was pilot tested on three students out of the sample for the validity of the tool. The interview questions were reduced from 12 items to 9 after a pilot study with some language changes.

\section{Data Analysis}

The researcher transcribed all the six interviews and conducted an inductive analysis. Themes and patterns were derived from interview responses of major research questions and probes through open coding. The voices of tenth class students were presented and discussed under each research question. The data was analysed manually

Table: Interview summary of grade $10^{\text {th }}$ students

\begin{tabular}{|c|c|c|c|c|c|c|c|c|c|c|c|}
\hline $\begin{array}{l}\mathrm{S} \\
\mathrm{T} \\
\mathrm{U} \\
\mathrm{D} \\
\mathrm{E} \\
\mathrm{N} \\
\mathrm{T} \\
\mathrm{S}\end{array}$ & $\begin{array}{l}\text { Favo } \\
\text { rite } \\
\text { subje } \\
\text { ct }\end{array}$ & $\begin{array}{l}\text { Views } \\
\text { about } \\
\text { mathem } \\
\text { atics }\end{array}$ & $\begin{array}{l}\text { Remarks } \\
\text { about } \\
\text { mathema } \\
\text { tics book }\end{array}$ & $\begin{array}{l}\text { Course } \\
\text { materi } \\
\text { als \& } \\
\text { your } \\
\text { underst } \\
\text { anding } \\
\text { level }\end{array}$ & $\begin{array}{l}\text { Your } \\
\text { thinking } \\
\text { about } \\
\text { the } \\
\text { nature } \\
\text { of } \\
\text { mathem } \\
\text { atics }\end{array}$ & $\begin{array}{l}\text { Uses } \\
\text { of } \\
\text { mathe } \\
\text { matics } \\
\& \\
\text { people } \\
\text { beliefs } \\
\text { around } \\
\text { you }\end{array}$ & $\begin{array}{l}\text { You are } \\
\text { good } \\
\text { mathem } \\
\text { atician }\end{array}$ & $\begin{array}{l}\text { Your } \\
\text { interest } \\
\text {, way } \\
\text { of } \\
\text { learnin } \\
\mathrm{g} \quad \& \\
\text { approa } \\
\text { ches }\end{array}$ & $\begin{array}{l}\text { Your } \\
\text { views } \\
\text { about } \\
\text { your } \\
\text { mathema } \\
\text { tics } \\
\text { teacher }\end{array}$ & $\begin{array}{l}\text { way of } \\
\text { teaching } \\
\text { students } \\
\text { particip } \\
\text { ation } \\
\text { and } \\
\text { students } \\
\text { involve } \\
\text { ment }\end{array}$ & $\begin{array}{l}\text { your } \\
\text { mathemati } \\
\text { cs } \\
\text { classroom } \\
\text { scenario }\end{array}$ \\
\hline
\end{tabular}




\begin{tabular}{|c|c|c|c|c|c|c|c|c|c|c|c|}
\hline $\mathrm{R} 1$ & Urdu & $\begin{array}{l}\text { Dislike, } \\
\text { don't } \\
\text { underst } \\
\text { and, } \\
\text { difficult }\end{array}$ & $\begin{array}{l}\text { Dislike } \\
\text { whole } \\
\text { book } \\
\text { except } \\
\text { chapter } \\
\text { five }\end{array}$ & $\begin{array}{l}\text { Not } \\
\text { satisfie } \\
\text { d with } \\
\text { the } \\
\text { course } \\
\text { materi } \\
\text { al, } \\
\text { very } \\
\text { low }\end{array}$ & $\begin{array}{l}\text { Good } \\
\text { subject } \\
\text { but } \\
\text { boring, } \\
\text { concept } \\
\text { ual }\end{array}$ & $\begin{array}{l}\text { Use in } \\
\text { daily } \\
\text { life, } \\
\text { sister- } \\
\text { like, } \\
\text { my } \\
\text { concep } \\
\text { tual all } \\
\text { friends } \\
\text { dislike }\end{array}$ & $\begin{array}{l}\text { No, try } \\
\text { to solve } \\
\text { problem } \\
\text { s but } \\
\text { failed }\end{array}$ & $\begin{array}{l}\text { Very } \\
\text { Low } \\
\text { interest } \\
\text {, take } \\
\text { guidan } \\
\text { ce for } \\
\text { learnin } \\
\mathrm{g}\end{array}$ & $\begin{array}{l}\text { I like her, } \\
\text { rout } \\
\text { behavior, } \\
\text { not } \\
\text { regular, }\end{array}$ & $\begin{array}{l}\text { Good, } \\
\text { lecture } \\
\text { method. } \\
15 \text { out } \\
\text { of } 94\end{array}$ & $\begin{array}{l}\text { Dislike } \\
\text { mathemati } \\
\text { cs class, } \\
\text { feel } \\
\text { hesitation } \\
\text { due to not } \\
\text { understan } \\
\text { ding }\end{array}$ \\
\hline $\mathrm{R} 2$ & $\begin{array}{l}\text { Engl } \\
\text { ish }\end{array}$ & $\begin{array}{l}\text { Like, } \\
\text { formula } \\
\text { is very } \\
\text { importa } \\
\text { nt for } \\
\text { underst } \\
\text { anding, } \\
\text { increase } \\
\text { mental } \\
\text { ability }\end{array}$ & $\begin{array}{l}\text { Like the } \\
\text { whole } \\
\text { book } \\
\text { because } \\
\text { it is } \\
\text { easier } \\
\text { than } \\
\text { other } \\
\text { science } \\
\text { subjects }\end{array}$ & $\begin{array}{l}\text { satisfie } \\
\text { d with } \\
\text { the } \\
\text { course } \\
\text { materi } \\
\text { al, } \\
\text { excelle } \\
\text { nt }\end{array}$ & $\begin{array}{l}\text { Good } \\
\text { subject, } \\
\text { interesti } \\
\text { ng, } \\
\text { concept } \\
\text { ual }\end{array}$ & $\begin{array}{l}\text { Mostly } \\
\text { use in } \\
\text { daily } \\
\text { life } \\
\text { especia } \\
\text { lly for } \\
\text { calcula } \\
\text { tion, } \\
\text { brother } \\
\text { like\& } \\
\text { sister } \\
\text { dislike, } \\
\text { three } \\
\text { friends } \\
\text { dislike } \\
\text { one } \\
\text { like }\end{array}$ & Yes & $\begin{array}{l}\text { High, } \\
\text { look to } \\
\text { the } \\
\text { formul } \\
\text { a \& \& } \\
\text { then } \\
\text { solve } \\
\text { by self, } \\
\text { mostly } \\
\text { solve } \\
\text { throug } \\
\text { h } \\
\text { exampl } \\
\text { e then } \\
\text { check } \\
\text { the } \\
\text { answer }\end{array}$ & $\begin{array}{l}\text { Like very } \\
\text { much, } \\
\text { punctual, } \\
\text { strict, } \\
\text { very } \\
\text { busy } \\
\text { therefore } \\
\text { not } \\
\text { regular, } \\
\text { confident } \\
\text { comman } \\
\text { d over } \\
\text { the } \\
\text { subject }\end{array}$ & $\begin{array}{l}\text { Very } \\
\text { good, } \\
\text { lecture } \\
\text { method, } \\
10 \quad \text { out } \\
\text { of } 94 \\
\text { students }\end{array}$ & $\begin{array}{l}\text { Like } \\
\text { mathemati } \\
\text { cs class, } \\
\text { feel very } \\
\text { happy } \\
\text { when } \\
\text { learning } \\
\text { something } \\
\text { new }\end{array}$ \\
\hline R3 & $\begin{array}{l}\text { Biol } \\
\text { ogy }\end{array}$ & $\begin{array}{l}\text { Mediu } \\
\mathrm{m} \text { Like } \\
\text { because } \\
\text { it is a } \\
\text { part of } \\
\text { educati } \\
\text { on, very } \\
\text { difficult }\end{array}$ & $\begin{array}{l}\text { Like } \\
\text { book if } \\
\text { understa } \\
\text { nd }\end{array}$ & $\begin{array}{l}50 \% \\
\text { satisfie } \\
\mathrm{d} \text {, if } \\
\text { teach } \\
\text { politel } \\
y \text { then } \\
\text { underst } \\
\text { anding } \\
\text { level is } \\
\text { good }\end{array}$ & $\begin{array}{l}\text { Good } \\
\text { subject, } \\
\text { interesti } \\
\text { ng when } \\
\text { understa } \\
\text { nd } \\
\text { otherwi } \\
\text { se } \\
\text { boring, } \\
\text { concept } \\
\text { ual }\end{array}$ & $\begin{array}{l}\text { Use in } \\
\text { daily } \\
\text { life, } \\
\text { my } \\
\text { father } \\
\text { like it, } \\
\text { brother } \\
\text { like \& } \\
\text { sister } \\
\text { dislike, } \\
\text { some } \\
\text { friend } \\
\text { like } \\
\text { some } \\
\text { dislike }\end{array}$ & $\begin{array}{l}\text { A little } \\
\text { bit } \\
\text { good, } \\
\text { non- } \\
\text { good } \\
\text { problem } \\
\text { solver }\end{array}$ & $\begin{array}{l}\text { Mediu } \\
\mathrm{m} \\
\text { interest } \\
\text {, take } \\
\text { guidan } \\
\text { ce for } \\
\text { solving } \\
\text { proble } \\
\text { ms }\end{array}$ & $\begin{array}{l}\text { I Like } \\
\text { her, } \\
\text { Good } \\
\text { teacher, } \\
\text { very } \\
\text { strict, } \\
\text { confident } \\
\text { ' } \\
\text { comman } \\
\text { d over } \\
\text { the } \\
\text { subject }\end{array}$ & $\begin{array}{l}\text { First } \\
\text { solve } \\
\text { example } \\
\text { students } \\
\text { respons } \\
\text { e eight } \\
\text { out of } \\
94,\end{array}$ & $\begin{array}{l}\text { Like } \\
\text { mathemati } \\
\text { cs class, } \\
\text { well- } \\
\text { motivated } \\
\text { but not } \\
\text { very good } \\
\text { in solving } \\
\text { questions. }\end{array}$ \\
\hline $\mathrm{R} 4$ & Urdu & $\begin{array}{l}\text { Dislike, } \\
\text { don't } \\
\text { underst } \\
\text { and, } \\
\text { very } \\
\text { difficult }\end{array}$ & $\begin{array}{l}\text { Dislike if } \\
\text { I leave } \\
\text { all } \\
\text { subject } \\
\text { and try to } \\
\text { learn } \\
\text { mathema } \\
\text { tics I will } \\
\text { not } \\
\text { understa } \\
\text { nd }\end{array}$ & $\begin{array}{l}\text { Not } \\
\text { satisfie } \\
\text { d with } \\
\text { the } \\
\text { course } \\
\text { materi } \\
\text { al, } \\
\text { very } \\
\text { low, I } \\
\text { have } \\
\text { no } \\
\text { interest }\end{array}$ & $\begin{array}{l}\text { Bad } \\
\text { subject }\end{array}$ & $\begin{array}{l}\text { Not } \\
\text { use in } \\
\text { daily } \\
\text { life, } \\
\text { my } \\
\text { parents } \\
\text { dislike } \\
\text { it, } \\
\text { brother } \\
\text { like \& } \\
\text { sister } \\
\text { dislike, } \\
\text { all } \\
\text { friends } \\
\text { dislike }\end{array}$ & No, & $\begin{array}{l}\text { Low } \\
\text { interest } \\
, \quad \text { take } \\
\text { guidan } \\
\text { ce, }\end{array}$ & $\begin{array}{l}\text { Yes I } \\
\text { like Rout } \\
\text { behavior, } \\
\text { punctual, } \\
\text { not } \\
\text { regular } \\
\text { Strict, } \\
\text { confident }\end{array}$ & $\begin{array}{l}\text { A good } \\
\text { way of } \\
\text { teaching } \\
, \quad 10 \\
\text { students } \\
\text { out of } \\
94\end{array}$ & $\begin{array}{l}\text { Don't like } \\
\text { mathemati } \\
\text { cs class, } \\
\text { feel } \\
\text { sadness } \\
\text { because I } \\
\text { not } \\
\text { understan } \\
\text { d }\end{array}$ \\
\hline R5 & Urdu & $\begin{array}{l}\text { At } \\
\text { primary } \\
\text { level }\end{array}$ & $\begin{array}{l}\text { Dislike } \\
\text { book } \\
\text { specially }\end{array}$ & $\begin{array}{l}\text { Not } \\
\text { satisfie } \\
\text { d }\end{array}$ & $\begin{array}{l}\text { Boring, } \\
\text { difficult } \\
, \quad \text { bad }\end{array}$ & $\begin{array}{l}\text { Use in } \\
\text { daily } \\
\text { life, }\end{array}$ & $\begin{array}{l}\text { No and } \\
\text { never }\end{array}$ & $\begin{array}{l}\text { Very } \\
\text { low, } \\
\text { no }\end{array}$ & $\begin{array}{l}\text { Like but } \\
\text { don't like } \\
\text { her }\end{array}$ & $\begin{array}{l}\text { Good,1 } \\
1 \\
\text { students }\end{array}$ & $\begin{array}{l}\text { Not like } \\
\text { be very } \\
\text { sad about }\end{array}$ \\
\hline
\end{tabular}




\begin{tabular}{|c|c|c|c|c|c|c|c|c|c|c|c|}
\hline & & $\begin{array}{l}\text { like } \\
\text { now } \\
\text { Extrem } \\
\text { ely } \\
\text { dislike, }\end{array}$ & $\begin{array}{l}\text { theorem, } \\
\text { like only } \\
\text { factorizat } \\
\text { ion }\end{array}$ & & $\begin{array}{l}\text { subject, } \\
\text { rata } \\
\text { subject } \\
\text { should } \\
\text { be } \\
\text { remove } \\
d \text { from } \\
\text { the } \\
\text { course }\end{array}$ & $\begin{array}{l}\text { my } \\
\text { sister } \\
\text { and } \\
\text { brother } \\
\mathrm{s} \\
\text { friends } \\
\text { like it }\end{array}$ & & $\begin{array}{l}\text { guidan } \\
\text { ce } \\
\text { taking } \\
\text { and try } \\
\text { to } \\
\text { solve } \\
\text { the } \\
\text { proble } \\
\text { m }\end{array}$ & $\begin{array}{l}\text { presence } \\
\text { in the } \\
\text { class, } \\
\text { very } \\
\text { confident } \\
\text {, }\end{array}$ & $\begin{array}{l}\text { give, } \\
\text { respons } \\
\text { e out of } \\
94\end{array}$ & $\begin{array}{l}\text { starting } \\
\text { class, } \\
\text { don't take } \\
\text { interest }\end{array}$ \\
\hline R 6 & $\begin{array}{l}\text { Engl } \\
\text { ish }\end{array}$ & $\begin{array}{l}\text { Like, I } \\
\text { underst } \\
\text { and, } \\
\text { very } \\
\text { easy } \\
\text { subject }\end{array}$ & $\begin{array}{l}\text { Like } \\
\text { mathema } \\
\text { tics book } \\
\text { only } \\
\text { theorem } \\
\text { dislike }\end{array}$ & $\begin{array}{l}\text { Satisfi } \\
\text { ed. } \\
\text { Unders } \\
\text { tandin } \\
\mathrm{g} \text { level } \\
\text { is very } \\
\text { high } \\
\text { and } \\
\text { take } \\
\text { more } \\
\text { interest }\end{array}$ & $\begin{array}{l}\text { Very } \\
\text { easy } \\
\text { subject, } \\
\text { interesti } \\
\text { ng, } \\
\text { good } \\
\text { subject }\end{array}$ & $\begin{array}{l}\text { Brothe } \\
\mathrm{r} \text { like } \\
\text { and } \\
\text { sister } \\
\text { don't } \\
\text { like, } \\
\text { friends } \\
\text { dislike } \\
\text { only } \\
\text { one } \\
\text { friend } \\
\text { like it }\end{array}$ & Yes & $\begin{array}{l}\text { Very } \\
\text { high, } \\
\text { solve } \\
\text { by } \\
\text { itself, } \\
\text { throug } \\
\text { h } \\
\text { practic } \\
\text { e I } \\
\text { learn } \\
\text { more }\end{array}$ & $\begin{array}{l}\text { I like her } \\
\text { very } \\
\text { much, } \\
\text { comman } \\
\text { d over } \\
\text { the } \\
\text { subject, } \\
\text { punctual, } \\
\text { not } \\
\text { regular }\end{array}$ & $\begin{array}{l}\text { Very } \\
\text { good, } \\
\text { only } 20 \\
\text { students } \\
\text { give } \\
\text { respons } \\
\text { e out of } \\
94\end{array}$ & $\begin{array}{l}\text { Yes I like } \\
\text { feeling } \\
\text { happy } \\
\text { when } \\
\text { mathemati } \\
\text { cs class is } \\
\text { started, } \\
\text { actively } \\
\text { participate } \\
\text { I I am } \\
\text { group } \\
\text { leader and } \\
\text { solve } \\
\text { question } \\
\text { for the } \\
\text { students }\end{array}$ \\
\hline
\end{tabular}

\section{Results}

For maintaining the ambiguity of respondents, the interviews were given codes as R1, R2 for respondent one, and respondent two respectively. The responses and the interpretation is given below: Q.1 which subject do you like in your school subject express your view about Mathematics? The thematic analysis of responses for the first question revealed that R1, R4, and R5 like Urdu while R2, R6 like English and R3 like biology in their school subjects. The beliefs of R1 and R4 were the same was they said that they dislike mathematics because it is very difficult and they could not understand very well. R2 said that she like mathematics because it increased mental ability. She beliefs that learning mathematics formula is very important.R3 expresses her views that she likes mathematics 50\% because it is a part of education. 50\% dislike because of difficulty. R5 extremely dislikes mathematics due to her frailer in the previous class.

\section{Q2. How is mathematics textbook and course material for you and which chapter you like or dislike?}

The response for the third and fourth questions exposed their beliefs about the book and course material of mathematics that is R1 and R4 have the same beliefs they dislike the book of mathematics and not satisfied with the course materials of mathematics. R2 likes the book of mathematics and satisfied with course materials and said that mathematics is easier than the other science subjects while R3 was 50\% satisfied and said that if she understands the course then she like it. R5 also dislikes the book of mathematics and not satisfied with the course materials she further explained that she liked only factorization in the book. R6 expresses her view about the book and the course materials that she like the book only dislike theorem. She was satisfied with the course materials and her understanding level is very high, take more interest.

\section{Q3. Express your thinking about the nature of mathematics?}

Responses for this question were that R1 expresses her view about the nature of mathematics she said that it is a good subject bur for me it is boring I am not taking interesting in this subject it is a conceptual subject and this subject should be optional.R2 revealed her beliefs in such a way that mathematics is a first-class and interesting subject further she explained that it is a conceptual subject and should be compulsory. R3 presented her belief that if she understands then it is very interesting for me and a very good subject. When sometime I am unable to understand then it is become boring for me and in my opinion it is not a memorizing subject. R4 said that it is a very terrible subject I dislike it.R5 responded against this question that mathematics is difficult, dull, and rata subject this 
subject should be removed from the course.R6 presented her view that it is very easy because if a person understands one question then can solve the whole exercise it is very interesting I enjoy very much when I solve the question and it is a superior subject.

Q.4 Say a few sentences about the uses of mathematics in your daily life and people beliefs about mathematics around you?

The thematic analysis of responses for this question exposed that R1 said that although I dislike mathematics but in reality its use is very much in our daily life all my friends dislike mathematics just like me. R2 revealed that this subject mostly used in our daily life especially for calculation in my home my brother likes it but my sister dislikes it I have four friends in which one likes it and the rest of the three dislike it. R3 beliefs about mathematics is that it is a good subject and use daily life furthermore she explained that my father and brother like it and my sister dislike my some friends like and some dislike.R4 strongly dislike this subject, therefore, she claimed that it is not used in our daily life and my parents, brother, sister and my friends dislike mathematics. R5 bare beliefs about mathematics were that this subject used in our daily life and my parents, brother, sister, and friends like it. R6 possessed view that it is used in every walk of life without mathematics life is incomplete furthermore she explained that her brother and friends like it only her sister and one friend dislike mathematics.

\section{Q5. You are a good mathematician?}

Responses for this question was very simple. R2 and R6 have the same view they claimed that they are a good mathematician and can solve problems in a very good manner. R1 and R4 possessed the same views that they are not a good mathematician. They said that they try more for solving problem but failed. R3 responses were average she said that she is a little bit good in mathematics and no good problem solver.R5 responded against this question that she is not a good mathematician and future she has no hope for this.

Q6. Being a learner of mathematics share your interest, your way of learning of mathematics? For the answer of six questions the responses were for R1 her that she did not like this subject therefore her interest is very low towards this subject. She said that she is very weak in this subject that is why she takes guidance from others for learning mathematics. R2 views were very positive about mathematics, therefore, her interest was very high she explained that for learning mathematics first, she looks to the formula \& then solve by self, mostly solve through example then check the answer for satisfaction.R3 responses for this question was normal not very high and not very low she said that she takes guidance for solving the problem. R4 answers to this question was that she said that her interest is very low and she take guidance for learning mathematics. R5 responses to this question was very weak the respondent said that she has no interest in learning mathematics and she is not trying to solve problems therefore she does not need guidance. Besides she said that she wrote memorized the question along with their answer for successes in the examination. R6 reacts positively she believed in practice she said that through practice she learn much more. She solved questions by itself and keep a high interest in mathematics.

Q7. Say a few sentences about your mathematics teacher?

For the answer of seven questions, almost all of the respondents had the same opinion for some points about their mathematics teacher. All of them like their mathematics teacher. All the respondents agree that their teacher is punctual but not regular and her behavior is very rout. R1 said that our teacher is very strict due to her strictness I dislike mathematics. R2 replies was that our teacher is good and has a good command over the subject. R3 expresses her view that our teacher is very confident but very busy therefore not completed her course on time.R4 and R6 said that our teacher is very confident and deliver information through lecture method. R5 response was very strange because she said that she likes her teacher but don't like her presence in the class due to mathematics anxiety.

Q 8. Share your teacher way of teaching and students involved during the lecture?

Responding to this question, the respondents stated their views about their teacher method all the respondents agreed that their teacher used a lecture method for solving problems. Further, all of them agree on the point that she is a good teacher but she solves examples only, and then we solve the whole exercise by self for which the interested students used key/guide and rest of the students leave it unsolved. According to all the interviewee students' involvement range is from 10 to 15 out of 94 students which is very low and UN satisfaction. 


\section{Q.9 would you like to share your mathematics classroom scenario?}

The thematic analysis of responses for this question was revealed in the classroom environment.R1 exposed that she dislikes mathematics classroom. She said that when a bell rings for mathematics she becomes very upset and feels hesitation due to not understanding. R2 gives responses that she likes the mathematics classroom and feels very happy especially when learning something new. R3 communicates her views that she is well motivated towards mathematics classroom but not very good at solving problem. R4 takes action against this question that she doesn't like mathematics classroom and feel sadness due to non-understanding. R5 said that she does not like mathematics class due to not understanding and not taking an interest in the class. R6 replied for this question that she like mathematics and feel very happy when mathematics class is started, she is an active participant, I am group leader and solve question for the students.

\section{Conclusion}

It is concluded from this investigation that at school mathematics is not preferred for the first category. Those students who understand the concept of mathematics possessed the positive beliefs about mathematics they also like the book and course materials and satisfied with it. They feel easy during mathematics classrooms, enjoy, and excited to learn something new in this subject. In contrast, the learner, who do not understand level is low they hold negative beliefs they did not like the book and course materials and not satisfied and feel hesitation during mathematics classroom.

The learners who have positive beliefs about mathematics, think mathematics is a good easy, interesting, and conceptual subject and should be compulsory at all levels because it is used in our daily life. The learner who have negative beliefs about mathematics they think that it is a difficult, boring and bad subject they think that it should be an optional subject at all level including secondary classes. They also said that there is no importance of mathematics in our daily life.

Optimistic believer of mathematics like their mathematics teacher, enjoy her presence in the classroom, and possess positive expression for her. Unenthusiastic believers like her teacher but don't like her presence in the classroom and feel hesitation during the lecture.

The researcher also concluded that in the construction of beliefs that are positive or negative about the mathematics classroom environment play a great role especially mathematics teacher, her methods, and her beliefs. If the teacher involves the students in activities, keeps a good and interesting method, and motivates the students from time to time then the learner will possess positive beliefs and will take interest in such a very important subject.

\section{References}

Akhter, N. (2018). Learning in Mathematics: Difficulties and Perceptions of Students. Journal of Educational Research, Dept. of Education, IUB, Pakistan (Vol. 21 No. 1).Pp 148, 149

Aksu, M. (2012). Students' Beliefs about Mathematics: A Descriptive Study

Ali, T. (2011). Exploring students' learning difficulties in a secondary mathematics classroom in Gigli-Baltistan and teachers' effort to help students overcome these difficulties. Bulletin of Education and Research, 33(1), 47-69.

Armianti. (2009). Komunikasi Matematika dan Kecerdasan Emosional. Prosiding Seminar Nasional Matematika dan Pendidikan Matematika Jurusan Pendidikan Matematika

FMIPA UNY, 5, 270-280. Retrieved from http://eprints.uny.ac.id/7030/1/P16-Armiati.pdf

Beswick, K. (2011). Knowledge/ beliefs and their relationship to emotion. In K. Kislenko (Ed.), Current state of research on mathematical beliefs XVI: Proceedings of the MAVI-16 conference June 26-29, 2010 (pp.43-59). Institute of Mathematics and Natural Sciences, Tallinn University, Estonia.

Bishop, A. J. (1997). The relationship between mathematics education and culture. In Iranian Mathematics Education Conference.

Callejo, M. L., \& Vila, A. (2009). Approach to mathematical problem solving and studentse

Belief systems: two case studies. Educational studies in mathematics, vol 72, and pp.111-126.

Chirove, M. (2014). "Relationship between learners' mathematically-related belief systems and their approach to mathematical problem solving: A case study of three High Schools in Tshwane North District (D3), South Africa" 77-81,

Ernest, P. (1989). The impact of beliefs on the teaching of mathematics. Mathematics teaching: The state of the art, 249, 254. http://webdoc.sub.gwdg.de/edoc/e/ pome/impact.htm. 
Furinghetti, F. \& Pehkonen, E. (2000). A comparative study of students' beliefs concerning their autonomy in doing mathematics. NOMAD 8(4), 7-26

Ferlinghetti, F., \& Pehkonen, E. (2002). Rethinking characterizations of beliefs. In G.C. Leder, E. Pehkonen, \& G. Torner (Eds). Beliefs: A hidden variable in mathematics education? 39-57. Dordrecht: Kluwer Academic Publishers.

Giovanni, P., \& Sangcap, A. (2010). Mathematics-related beliefs of Filipino college students:

Factors affecting mathematics and problem-solving performance. Procedia Social and Behavioral Sciences, 8, 466-475.www.sciencedirect.com

Goldin, G. A. (2002). Affect, meta-affect, and mathematical belief structures. In G. Leder, E. Pehkonen, \& G. Torner (Eds.). Beliefs: A hidden variable in mathematics education? 59-72. Dordrecht: Kluwer Academic Publishers.

Hadi, S. (2017). Realistic Mathematics Education: Theory of Development and Implementation. Jakarta: PT Raja Grafindo Persada. https://www.researchgate.net/publication/331532255

Hannula, M. S. (2007). The effect of achievement, gender, and classroom contexts on upper secondary students' mathematical beliefs. Lyon France.

Kıbrıslıoğlu, N., \& Haser, C. (2016). Development of mathematics-related belief scale for the 5thgrade students in Turkey. Retrieved on 12 January, 2020. https://hal.archives-ouvertes.fr/hal01287345

Kupari, P., \& Nissinen, K. (2013). Background factors behind mathematics achievement in Finnish education context: London: Routledge.

Levy, S. R., West, T., \& Rosenthal, L. (2012). The contributing role of prevalent belief systems to intergroup attitudes and behaviors. Online readings in psychology and culture Retrieved on January, 92020.

Maiorca, C. (2016). A Case study: Students Mathematics Related beliefs From Integrated STEM Model Electing Activities: Ph.D. thesis dissertation Pp 39,40.

Merriam, S. B. (1988). Case study research in education: A qualitative approach. San Francisco: Jossey-Bass Publisher.

Op't Eynde, P., De Corte, E. \& Verschaffel, L. (2002). Framing mathematics-related beliefs. In G Leder, E. Pehkonen, \& G. Törner (Eds.), Beliefs: A hidden variable in mathematics education (pp. 13-37). Boston, MA: Kluwer Academic Publishers.

Op"t Eynde, P. \& Hannula, M. S. (2006). The case study of Frank. Educational studies in mathematics (2006) 63: 123-129 DOI: 10.1007/s10649-006- 9030-8

Opt Eynde, P., De Corte, E., \& Verschaffel, L. (2006). Beliefs and metacognition: An analysis of Junior High School students' mathematics-related beliefs. In De Corte, A., \& Veenman, M. Metacognition in mathematics education. New York: Nova 1 Publishers.

Physick, M. D. (2010). Exploring mathematics-related belief systems (Doctoral dissertation, Faculty of Education-Simon Fraser University).

Schoenfeld, A. H. (2015). What counts, when? Reflection on beliefs, affect, attitude, orientations, habits of mind, grain size, time scale, context, theory, and method. From beliefs to dynamic affect systems in mathematics education, 395-404.

Smith, K, (2014). How Teacher Beliefs About Mathematics Affect Student Beliefs About Mathematic https:// scholar.unh.edu.honors.

Tambunan, H. (2014). Strategi Heuristik dalam Pemecahan Matematika Sekolah. Journal Saintech, 6(4), 3540. http://www.iejme.com

Tambunan, H. (2018). Impact of heuristic strategy on students' mathematics ability in high order thinking. International Electronic Journal of Mathematics Education, 13(3), 321-328.

Yuanita P, Zulnaidi. H, Zakaria E. (2018). The effectiveness of the Realistic Mathematics Education approach: The role of mathematical representation as mediators between mathematical belief and problem-solving. PLoS ONE 13(9): e0204847.https://doi.org/10.1371/journal. pone. 0204847 